\title{
Estimation Method of the Total Number of Wild Animals based on Modified Jolly's Method
}

\author{
Kohei Arai ${ }^{1}$ \\ ${ }^{1}$ Graduate School of Science and \\ Engineering \\ Saga University \\ Saga City, Japan
}

\author{
Takashi Higuchi ${ }^{1}$ \\ ${ }^{1}$ Graduate School of Science and \\ Engineering \\ Saga University \\ Saga City, Japan
}

\author{
Tetsuya Murakami ${ }^{2}$ \\ ${ }^{2}$ Fukuoka Agriculture and Forestry \\ Research Center \\ Yoshiki, Chikushino City, \\ Fukuoka, Japan
}

\begin{abstract}
Estimation method of the total number, the probabilities of birth and alive of wild animals based on Jolly's method is proposed. Jolly's method requires putting tags to the captured wild animals by bank trap while just identifications of the wild animals using camera images are required for the proposed method. An identification method is also proposed here. Other than these, the method for detection of specific wild animals is proposed. The proposed method is validated through simulations. The proposed method for specific wild animal detection with acquired camera images is also validated. The simulation results show that the proposed Modified Jolly's Method: MJM is superior to the conventional Petersen method by $2.65 \%$ in terms of confidence interval of the estimated total number of wild pigs in the simulation cells in concern (128 by 128).
\end{abstract}

Keywords-Wild animals; Jolly's method; Specific wild animal detection

\section{INTRODUCTION}

According to the West, B. C., A. L. Cooper, and J. B. Armstrong, 2009, "Managing wild pigs: A technical guide. Human-Wildlife Interactions Monograph", 1-551, there are the following wild pig damages, Ecological Impacts to ecosystems can take the form of decreased water quality, increased propagation of exotic plant species, increased soil erosion, modification of nutrient cycles, and damage to native plant species [1]-[5]. Agricultural Crops Wild pigs can damage timber, pastures, and, especially, agricultural crops [6]-[9]. Forest Restoration Seedlings of both hardwoods and pines, especially longleaf pines, are very susceptible to pig damage through direct consumption, rooting, and trampling [10]-[12]. Disease Threats to Humans and Livestock Wild pigs carry numerous parasites and diseases that potentially threaten the health of humans, livestock, and wildlife [13][15]. Humans can be infected by several of these, including diseases such as brucellosis, leptospirosis, salmonellosis, toxoplasmosis, sarcoptic mange, and trichinosis. Diseases of significance to livestock and other animals include pseudorabies, swine brucellosis, tuberculosis, vesicular stomatis, and classical swine fever [14], [16]-[18]. There are also some lethal techniques for damage managements. One of these is trapping. It is reported that an intense trapping program can reduce populations by 80 to $90 \%$ [19]. Some individuals, however, are resistant to trapping; thus, trapping

\footnotetext{
${ }^{1}$ www.berrymaninstitute.org/publications,
}

alone is unlikely to be successful in entirely eradicating populations. In general, cage traps, including both large corral traps and portable drop-gate traps, are most popular and effective, but success varies seasonally with the availability of natural food sources [20]. Cage or pen traps are based on a holding container with some type of a gate or door [21]. The method and system for monitoring the total number of wild pigs in the certain district in concern is proposed [22]. All the aforementioned system is not so cheap. It requires huge resources of human-ware, hardware and software as well. Also, it is totally time consumable task. Usually, it takes two years to finalize the total number of wild animals and wildlife damages. Therefore, it is hard to plan the countermeasures for the wildlife damages.

Wildlife damage in Japan is around 23 Billion Japanese Yen a year in accordance with the report from the Ministry of Agriculture, Japan. In particular, wildlife damages by deer and wild pigs are dominant (10 times much greater than the others) in comparison to the damage due to monkeys, bulbuls (birds), rats. Therefore, there are strong demands to mitigate the wildlife damage as much as we could. It, however, is not so easy to find and capture the wildlife due to lack of information about behavior. In particular, the total number, the probabilities of birth and alive of wild animals are not so easy to estimate even if a camera monitor is equipped.

The method for reducing the number of camera monitors based on Kriging method is proposed already [23]. Meanwhile, the prediction method of the total number of wild animals using blog and tweet information is also proposed so far [24]. It, however, still a problem to improve estimation and prediction accuracies.

Jolly's method allows to estimate the total number, the probabilities of birth and alive of wild animals through putting tags for the captured wild animals [25], [26]. It, however, is capable to identify specific wild animals by using acquired camera monitor images with the features of size, shape, face features. Therefore, there is no need to put tags to the captured wild animals for estimation of the total number, the probabilities of birth and alive of wild animals. It is called " Modified Jolly's Method: MJM" for estimation of the total On the other hand, it is capable to identify specific wild animals by using acquired camera monitor images with the features of size, shape, face features. Therefore, there is no need to put tags to the captured wild animals for estimation of the total 
number, the probabilities of birth and alive of wild animals.by using camera monitors. The number of camera monitors can be reduced by the previously proposed method based on Kriging method.

In this paper, the MJM is proposed together with the proposed method for specific wild animal identification. Then some simulations are followed by for validations of MJM and the specific wild animal identification. Finally, conclusion is described with some discussions.

\section{PROPOSED METHOD}

\section{A. Conventional Jolly's Method as well as Petersen Method}

Petersen proposed the following estimation method of total number of wild animals.

1) Capture the $k$ wild animals with traps and give them an identification sign. Released wild animals,

2) Once the wild animals were scattered to some extent in their area, once again, if you trap and capture the wild animals,

3) $c$ were caught in a trap. Among them, $m(<c)$ animals had attached identification tags

4) Then the total number of wild animals $M$ can be estimated with the following equation,

$M=m c / k$

The method can be extended as follows,

1) (1st stage) First, $c_{1}$ caught the wild animals in a certain area. And unleash the wild animals with an individual identification number.

2) (2 stages) When catching again after a certain period (thoroughly organism diffused), it was $c_{2}$. Among them, there were $m_{2}$ creatures with individual identification numbers. Also release newly captured wild animals with an individual identification number.

3) This was repeated, capturing $c_{\mathrm{i}}$ animals (stage i). Among them, that organism with an individual identification number was $m_{\mathrm{i}}$.

Let be the followings,

$N_{\mathrm{i}}$ : Total number of wild animals in i stage,

$M_{\mathrm{i}}$ : Total number of wild animals with individual identification number in stage $i$ (note that some wild animals died or came out of the range, so note that it is different from the total number of wild animals with individual identification numbers so far)

$R_{\mathrm{i}}$ : Number of items caught at least once after $c_{\mathrm{i}}$

$Z_{\mathrm{i}}$ : Number of individuals labeled before the $\mathrm{i}$-th time, number of captured objects that have not been captured at the i-th time and captured at least once thereafter

In this stage, since the probability of capturing again at the i-th time is the same as the rate at which the one having the identification number not captured at the $i$-th time is captured again,

$M_{\mathrm{i}}=m_{\mathrm{i}}+c_{\mathrm{i}} z_{\mathrm{i}} / r_{\mathrm{i}}$
Also, as we thought in the wild animals, the probability of choosing one with a sign should be the same from the probability of picking $c_{\mathrm{i}}$ from the total number of living wild animals $N_{\mathrm{i}}$ and the total number $M_{\mathrm{i}}$ of signs attached,

$m_{\mathrm{i}} / M_{\mathrm{i}}=c_{i} / N_{i}$

Therefore, the total number of living wild animals is expressed with the following equation,

$N_{i}=M_{\mathrm{i}} c_{i} / m_{\mathrm{i}}$

Next, let's look at the survival rate. It is the total number $M_{i}$ of marks attached at the i-th time, and those marked newly at the $\mathrm{i}$-th time are $c_{i}-. m_{i}$ It is $m_{i}$. Therefore, when all are alive, the total number $M_{i}+\left(c_{i}-. m_{i}\right)$ of markers attached at $\mathrm{i}$ +1 time. Actually it is $M_{\mathrm{i}+1}$, so the probability $p_{i}$ to survive is

$p_{i}=M_{\mathrm{i}+1}, /\left(M_{i}+\left(c_{i}-. m_{i}\right)\right)$

Next, let's look at the increment number (the number of births, the number of subscriptions). Given the interrogation interval as $t$ (day), given the multiplicative theorem of probability, $\mathrm{i}-1$, the survival rate between 1 and $\mathrm{i}$ times is represented as $p_{\mathrm{i}}^{\mathrm{t}}$.

Take into consideration that it will be born after 1 time or will enter from other than the subject of investigation and will die until $i$ or jump out of scope.

The probability that what was born for $k-1 / n$ and $k / n$ in $\mathrm{i}$ is alive can be expressed as follows,

$p_{\mathrm{i}}^{(1-\mathrm{k} / \mathrm{n})} / n$

Therefore, the probability of survival of what is born during this time is

$\sum_{\mathrm{k}=1}^{\mathrm{n}} p_{\mathrm{i}}^{(1-\mathrm{k} / \mathrm{n})} / n$

Considering the definition of integration (piecewise quadrature method),

$\sum_{k=1}^{n} p_{i}^{\left(1-\frac{k}{n}\right)} \frac{1}{n} \quad \stackrel{n \rightarrow \infty}{\longrightarrow} \quad \int_{0}^{1} p_{i}^{(1-t)} d t=\left[\frac{-e^{(1-t) \log p_{i}}}{\log p_{i}}\right]_{0}^{1}=\frac{p_{i}-1}{\log p_{i}}$

$\sqrt{p} \log p \fallingdotseq(p-1)$

To summarize the above, i. The probability that a thing born after the first time or entering from the other will survive to the $\mathrm{i}$ th survey is $\sqrt{p_{i}}$.

Therefore, for $\mathrm{i}$ in $\mathrm{i}-1$, the total number of subscribers obtained from observations between 1 and $i$ is

$N_{i}-p_{i-1} N_{\mathrm{i}-1}$

Let $B_{i}$ be the total number of subscriptions between 1 and $\mathrm{i}$. Thinking that the survival rate of newly born is the same as the survival rate $p_{i}$ at $\mathrm{i}$ times, $\mathrm{i}-1$ Born after one time, the number that survives i times is $\sqrt{ } p_{i} B_{i}$,

$\sqrt{ } p_{i} B_{i}=N_{i}-p_{i-1} N_{\mathrm{i}-1}$

Therefore, the total number of subscriptions between 1 and I can be expressed as follows,

$B_{i}=N_{i}-p_{i-1} N_{\mathrm{i}-1} /{ } p$

This is called "Jolly's Method". 


\section{B. Proposed Modified Jolly's Method}

There is no need to capture wild animals with traps for estimation of the total number of wild animals in the intensive areas. Instead of capturing wild animals, identification of specific wild animals is needed by using acquired camera images. Body size, limbs, ears, canines of the wild animals in concern are features extracted from the acquired images for identification. The proposed Modified Jolly's Method: MJM is based on the conventional Jolly's method for estimation of the total number of wild animals with the limited number of trials up to two times. Also, the identification of method for specific wild animals with acquired camera images is proposed.

\section{Proposed System Configuration}

Best ways are known mostly. On the ways, some network cameras are installed as shown in Fig.1. Wild animals are monitored with the near infrared video camera with near infrared Light Emission Diode: LED. Because wild pigs are active in nighttime, Near Infrared: NIR camera with NIR LED is used. Outlook of the NIR camera is shown in Fig.2 while the specification of the camera is shown in Table 1, respectively.

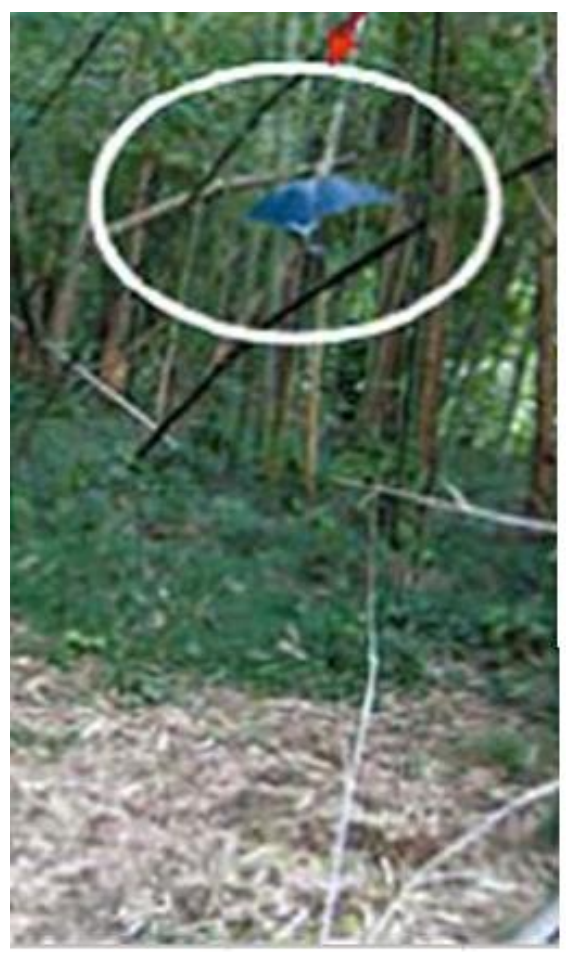

Fig. 1. Installed NIR camera (White circle)

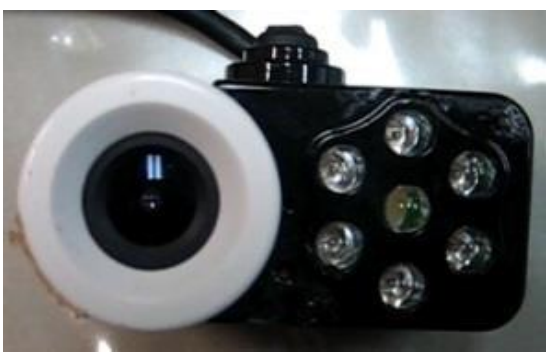

Fig. 2. Outlook of the NIR camera
TABLE I. SPECIFICATION OF NIR CAMERA (NETCOWBOY)

\begin{tabular}{|l|l|}
\hline Pixel & $1.3 \mathrm{M}$ \\
\hline Resolution & $1280 \times 1024$ \\
\hline \multirow{2}{*}{ Frame rate } & $1280 \times 1024: 7.5 \mathrm{fps}, 640 \mathrm{x}$ \\
& $480: 30 \mathrm{fps}$ \\
\hline \multirow{2}{*}{ Dimension } & $52 \mathrm{~mm}(\mathrm{~W}) \times 65 \mathrm{~mm}(\mathrm{D}) \times$ \\
\hline Weight & $70 \mathrm{~mm}(\mathrm{H})$ \\
\hline Operating condition & $85 \mathrm{~g}$ \\
\hline Interface & $0-40 \mathrm{deg} . \mathrm{C}$ \\
\hline IR Illumination & USB 2.0 \\
\hline & 7 \\
\hline
\end{tabular}

III. EXPERIMENTS

\section{A. Proposed Identification of Specific Wild Animals}

Moving pictures are acquired with high resolution mode of 1280 by 1024 pixels. Therefore, frame rate is $7.5 \mathrm{fps}$. OpenCV is used for acquisition, processing, and analysis because it is totally easy to use. OpenCV is an open source computer vision library which is written in $\mathrm{C}$ and $\mathrm{C}++$ and runs under Linux, Windows, and Mac OS X. It can be downloaded from http://sourceforge.net/projects/opencvlibrary

There so many library software for image processing and analysis. First, object has to be extracted from the moving picture. Then object contour has to be extracted. For the contour extraction and tracing, Canny filter related spatial filters are attempted. After that, it would be better to remove the background. The following background removals is attempted,

\section{cv2.createBackgroundSubtractorMOG()}

In order to discriminate female wild pigs, template matching method is applied with a template of small portion of nipple images. The following correlation functions are attempted for template matching,

\section{CV_TM_SQDIFF , CV_TM_SQDIFF_NORMED , CV_TM_CCORR , CV_TM_CCORR_NORMED , CV_TM_CCOEFF, CV_TM_CCOEFF_NORMED}

Also feature matching methods are applied for discrimination of female wild pigs. There are many feature matching methods in the OpenCV library. A couple of feature matching methods are attempted for the discriminations. The followings are typical feature matching methods which are provided from OpenCV,

- BruteForce

- BruteForce-L1

- BruteForce-SL2

- BruteForce-Hamming

- BruteForce-Hamming (2)

- FlannBased

The FlannBasedMatcher interface is used in the proposed method in order to perform a quick and efficient matching by using the FLANN (Fast Approximate Nearest Neighbor Search Library). Also Brute-Force matcher which is simple matching method is used in the proposed method. It takes the 
descriptor of one feature in first set and is matched with all other features in second set using some distance calculation. For both, feature descriptor is needed. Speeded-up Robust Feature: SURF is used in the proposed method.

One shot image of the acquired moving pictures is shown in Fig. 3 as an example. This is a female wild pig on the route from habitat area to go to the calms feed. Wild boar children are followed by the female wild pig. By using the difference between the current and the previous frame of wild pig (targeted object), it is possible to extract the female wild pig. Also, it is possible to remove the background by frame by frame. Fig.4 shows the resultant image of the background removals.

Edge and contour extractions are attempted with sharp Canny filters. Fig. 5 shows the resultant image of sharp Canny filter. Also, Fig.6 shows feature matching resultant image with FLANN with the nipples of the feature of the wild pig. Thus the specific features (size, nipple, limbs, ears, canines) of the wild pigs in concern can be extracted.

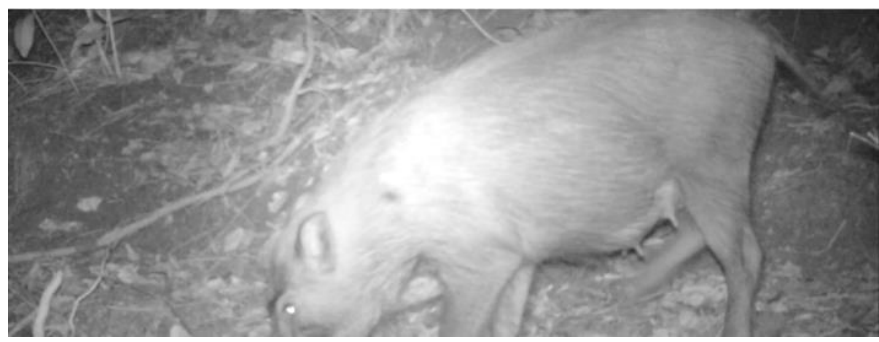

Fig. 3. Portion of original image of the targeted object of female wild pig in concern

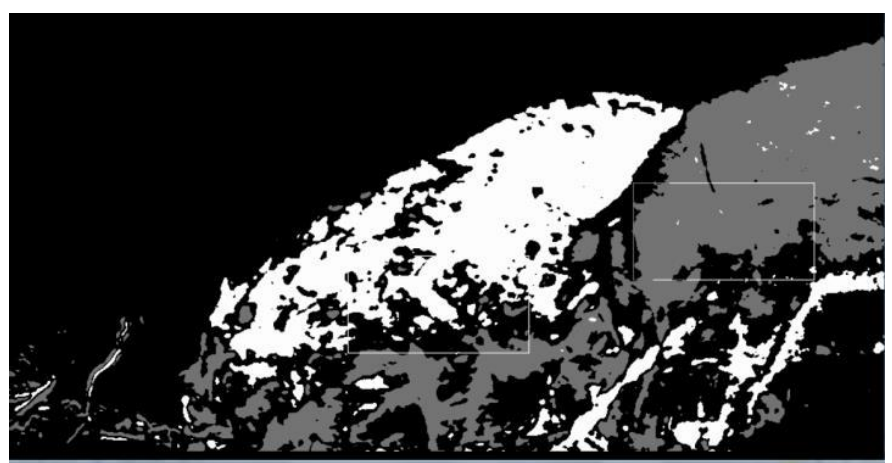

Fig. 4. Resultant image of background removal from the original image in frame by frame basis

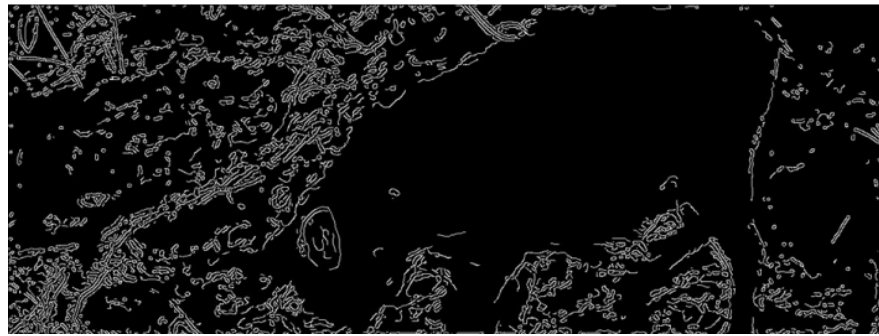

Fig. 5. Resultant images of edge and contour extractions by Sharp Canny filter

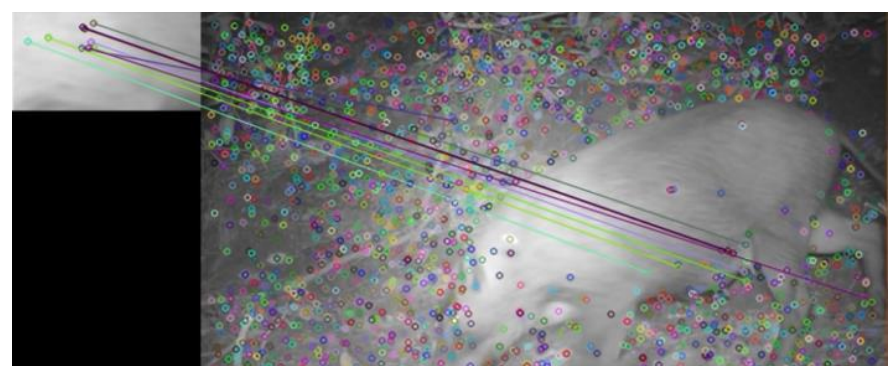

Fig. 6. Example of the resultant image of FLANN

\section{B. Simulation Study}

Simulation study is conducted. Wild animal route simulations are conducted with 128 by 128 cells. Wild animals move from one cell to the other cell. A portion of the simulation cells are shown in Fig.7. Original positions of wild animals are determined by random numbers. After that, wild animals move in accordance another random numbers. On the other hand, wild animal monitors are set on the designated cells regularly. Wild animal monitors are set at every cell in the first trial. Then the number of monitors is reduced by the factor of two. Namely, the monitors are set every two cells in the second trial and the monitors are set every four cells in the third trial and so on.

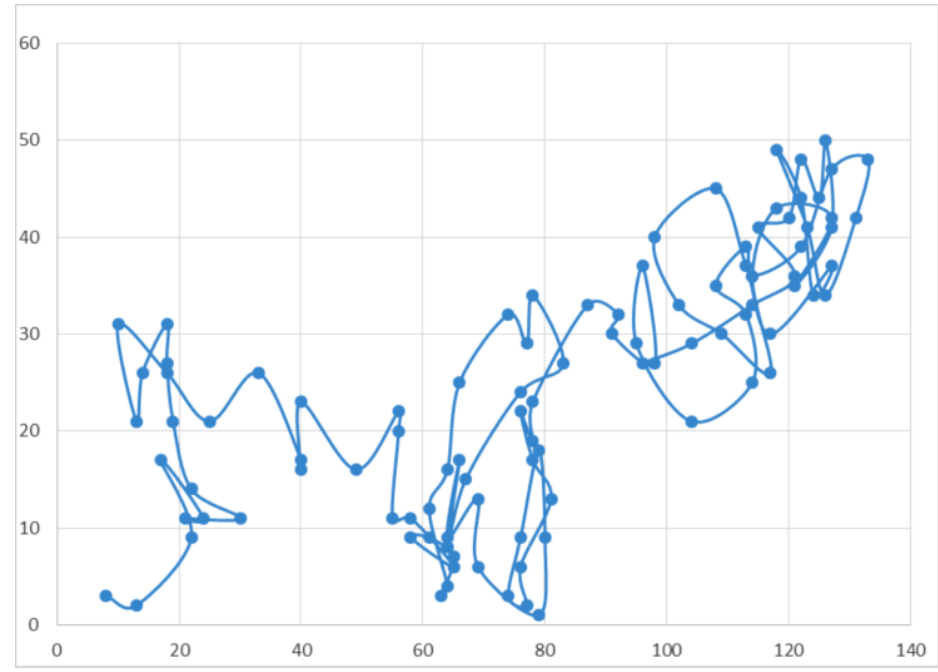

Fig. 7. Portion of simulation cells which consists of 128 by 128

If wild animal reach the cell which is supposed to be a wild animal monitor, then the number of captured wild animals is incremented. The simulation is conducted for 5000 trials with the total number of wild animals is set at 100 .

As the results of the simulations, it is found that the capture ratio recapturing ratio and the recapturing again ratio (captured for three times). For instance, the capture ratio of the wild pig \#1 is 0.3394 while recapturing ratio of the wild pig $\# 1$ is 0.1154 and the recapturing again ratio of the wild pig \#1 is 0.036 . Then the total number of wild pigs in the simulation cells is estimated with Pertersen method (using recapturing ratio) as 104.5264 in average while 26.9163 of standard deviation. On the other hand, the estimated total number of wild pigs by using the proposed MJM method (using 
recapturing again ratio) is 104.1191 in average with standard deviation of 27.6297.

Confident interval at the $95 \%$ of confidence level of the Persen method is 0.7461 as shown in Fig.8 (a) while that of the proposed MJM method is 0.7658 as shown in Fig.8 (b). $2.64 \%$ of improvement of the confidence interval is confirmed for the proposed MJM method in comparison to the conventional Petersen method.

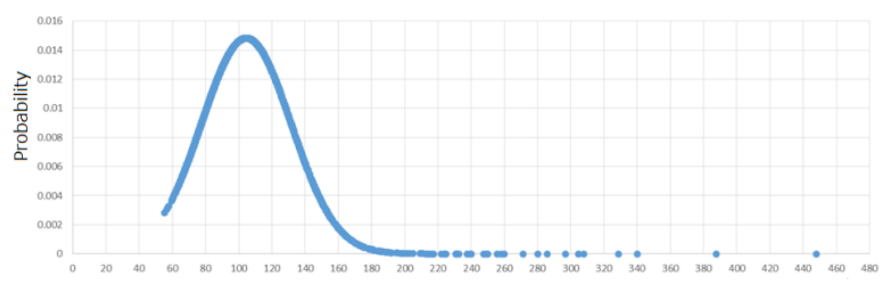

Estimated total number of wild pigs in the intensive simulation cells

(a) Petersen

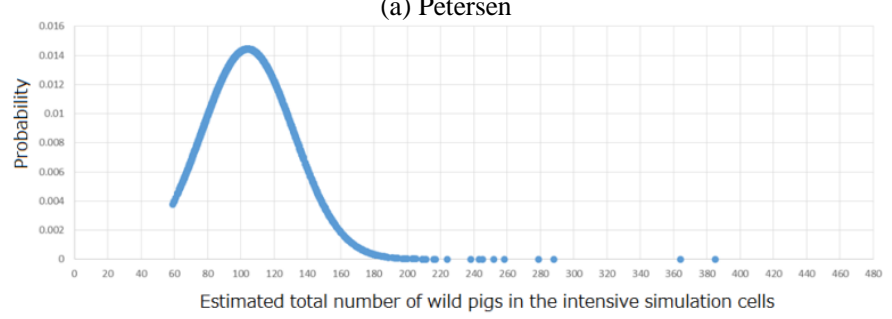

(b) Proposed MJM

Fig. 8. Probability density functions of the estimated total number of wild pigs in the simulation cells in concern

\section{CONCLUSION}

Estimation Method of the Total Number, the Probabilities of Birth and Alive of Wild Animals Based on Jolly's Method is proposed. The proposed method is validated through simulations. Also, the method for detect specific wild animals is proposed. The proposed method for specific wild animal detection with acquired camera images is also validated.

As the simulation results, it is found that the proposed Modified Jolly's Method: MJM is superior to the conventional Petersen method by $2.65 \%$ in terms of confidence interval of the estimated total number of wild pigs in the simulation cells in concern (128 by 128$)$.

Further investigation is required for improvement of specific wild animal identification accuracy through various feature extractions.

\section{ACKNOWLEDGEMENTS}

Author would like to thank the member of research group for wild animal damage mitigation lead by Mr. Tetsuya Murakami of Fukuoka Agriculture and Forestry Research Center for their valuable discussions throughout the research works and the experiments.

\section{REFERENCES}

[1] Patten, D. C. 1974. Feral hogs - boon or burden. Proceedings of the Sixth Vertebrate Pest Conference 6:210-234.

[2] Singer, F. J., W. T. Swank, and E. E. C. Clebsch. 1984. Effects of wild pig rooting in a deciduous forest. Journal of Wildlife Management. 48:464-473.
[3] Stone, C. P., and J. O. Keith. 1987. Control of feral ungulates and small mammals in Hawaii's national parks: research and management strategies. Pages $277-287$ in C. G. J. Richards and T. Y. Ku, editors. Control of mammal pests. Taylor and Francis, London, England, and New York and Philadelphia, USA.

[4] Cushman, J. H., T. A. Tierney, and J. M. Hinds. 2004. Variable effects of feral pig disturbances on native and exotic plants in a California grassland. Ecological Applications 14:1746-1756.

[5] Kaller, M. D., and W. E. Kelso. 2006. Swine activity alters invertebrate and microbial communities in a coastal plain watershed. American Midland Naturalist 156:163-177.

[6] Bratton, S. P. 1977. The effect of European wild boar on the flora of the Great Smoky Mountains National Park. Pages 47-52 in G. W. Wood, editor. Research and management of wild hog populations. Belle W. Baruch Forest Science Institute, Clemson University, Georgetown, South Carolina, USA.

[7] Lucas, E. G. 1977. Feral hogs - problems and control on National Forest lands. Pages 17-22 in G. W. Wood, editor. Research and management of wild hog populations. Belle Baruch Forest Science Institute, Clemson University, Georgetown, South Carolina, USA.

[8] Thompson, R. L. 1977. Feral hogs on National Wildlife Refuges. Pages 11-15 in G. W. Wood, editor. Research and management of wild hog populations. Belle W. Baruch Forest Science Institute, Clemson University, Georgetown, South Carolina, USA. Kohei Arai, Preliminary Assessment of Radiometric Accuracy for MOS-1 Sensors, International Journal of Remote Sensing, Vol.9, No.1, pp.5-12, Apr.1988.

[9] Schley, L, and T. J. Roper. 2003. Diet of wild boar Sus scrofa in Western Europe, with particular reference to consumption of agricultural crops. Mammal Review 33:43-56.

[10] Whitehouse, D. B. 1999. Impacts of feral hogs on corporate timberlands in the southeastern United States. Pages 108-110 in Proceedings of the Feral Swine Symposium, June 2-3, 1999, Ft. Worth, Texas, USA.

[11] Mayer, J. J., E. A. Nelson, and L. D. Wike. 2000. Selective depredation of planted hardwood seedlings by wild pigs in a wetland restoration area. Ecological Engineering, 15(Supplement 1): S79-S85.

[12] Campbell, T. A., and D. B. Long. 2009. Feral swine damage and damage management in forested ecosystems. Forest Ecology and Management 257:2319-2326

[13] Forrester, D. J. 1991. Parasites and diseases of wild mammals in Florida. University of Florida Press, Gainesville, Florida, USA.

[14] Williams, E. S., and I. K. Barker. 2001. Infectious diseases of wild mammals. Iowa State University Press, Ames, Iowa, USA.

[15] Sweeney, J. R., J. M. Sweeney, and S. W. Sweeney. 2003. Feral hog. Pages 1164-1179 in G. A. Feldhamer, B. C. Thompson, and J. A. Chapman, editors. Wild mammals of North America. Johns Hopkins University Press, Baltimore, Maryland, USA.

[16] Nettles, V.F., J. L. Corn, G. A. Erickson, and D. A. Jessup. 1989. A survey of wild swine in the United States for evidence of hog cholera. Journal of Wildlife Diseases 25:61-65.

[17] Davidson, W. R., and V. F. Nettles, editors. 1997. Wild swine. Pages 104-133 in Field manual of wildlife diseases in the southeastern United States. Second edition. Southeastern Cooperative Wildlife Disease Study, Athens, Georgia, USA.

[18] Davidson, W. R., editor. 2006. Wild swine. Pages 105-134 in Field manual of wildlife diseases in the southeastern United States. Third . Southeastern Cooperative Wildlife Disease Study, Athens, Georgia, USA.

[19] Choquenot, D. J., R. J. Kilgour, and B. S. Lukins. 1993. An evaluation of feral pig trapping. Wildlife Research, 20:15-22.

[20] Barrett, R. H., and G. H. Birmingham. 1994. Wild pigs. Pages D65-D70 in S. Hyngstrom, R. Timm, and G. Larsen, editors. Prevention and control of wildlife damage. Cooperative Extension Service, University of Nebraska, Lincoln, Nebraska, USA.

[21] Mapston, M. E. 1999. Feral hog control methods. Pages 117-120 in Proceedings of the Feral Swine Symposium, June 2-3, 1999, Fort Worth, Texas, USA

[22] Kohei Arai, Indra Nugraha Abdullah, Kensuke Kubo, Katsumi Sugaw, Methods for Wild Pig Identifications from Moving Pictures and 
Discrimination of Female Wild Pigs based on Feature Matching Method, (IJARAI) International Journal of Advanced Research in Artificial Intelligence, Vol. 4, No.7, 41-46, 2015

[23] Kohei Arai, Takashi Higuchi, method for Reducing the Number of Wild Animal Monitors by Means of Kriging, International Journal of Advanced Research on Artificial Intelligence, 5, 5, 14-20, 2016.

[24] Kohei Arai, Shohei Fujise, Wildlife Damage Estimated and Prediction Using Blog and Tweet Information, International Journal of Advanced Computer Science and Applications, 5, 4, 15-21, 2016.

[25] Seber, G.A.F. The Estimation of Animal Abundance and Related Parameters. Caldwel, New Jersey: Blackburn Press. ISBN 1930665555

[26] Krebs, C.J. 1998. Ecological methodology. 2nd Ed. Benjamin Cummings. Menlo Park, CA. 620p. ISBN 9780321021731

\section{AUTHORS PROFILE}

Kohei Arai, He received BS, MS and PhD degrees in 1972, 1974 and 1982, respectively. He was with The Institute for Industrial Science and Technology of the University of Tokyo from April 1974 to December 1978 and also was with National Space Development Agency of Japan from January, 1979 to March, 1990. During from 1985 to 1987, he was with Canada Centre for Remote Sensing as a Post-Doctoral Fellow of National Science and Engineering Research Council of Canada. He moved to Saga University as a Professor in Department of Information Science on April 1990. He was a counselor for the Aeronautics and Space related to the Technology Committee of the Ministry of Science and Technology during from 1998 to 2000. He was a councilor of Saga University for 2002 and 2003. $\mathrm{He}$ also was an executive councilor for the Remote Sensing Society of Japan for 2003 to 2005. He is an Adjunct Professor of University of Arizona, USA since 1998. He also is Vice Chairman of the Commission-A of ICSU/COSPAR since 2008. He received Science and Engineering Award of the year 2014 from the minister of the ministry of Science Education of Japan and also received the Bset Paper Award of the year 2012 of IJACSA from Science and Information Organization: SAI. In 2016, he also received Vikram Sarabhai Medal of ICSU/COSPAR and also received 37 awards. He wrote 37 books and published 570 journal papers as well as 370 conference papers. He is Editor-in-Chief of International Journal of Advanced Computer Science and Applications as well as International Journal of Intelligent Systems and Applications. http://teagis.ip.is.saga-u.ac.jp/ 